\title{
Stenting versus non-stenting before extracorporeal shock wave lithotripsy for proximal ureteric stones: A prospective interventional study
}

\author{
Vedamurthy Reddy Pogula', Sandeep Reddy ${ }^{2}$, Ershad Hussain Galeti ${ }^{3}$, Mustaq Rasool ${ }^{4}$ \\ ${ }^{1}$ Professor and Head, ${ }^{2,3,4}$ Senior Resident, Department of Urology, Narayana Medical College, Nellore, \\ Andhra Pradesh, India
}

A B S T R A C T

Background: Extracorporeal shock wave lithotripsy (ESWL) has become the first-line treatment for stones in the upper urinary tract measuring $<2 \mathrm{~cm}$. It is the minor invasive treatment for calculi of the upper urinary tract. Aims and Objectives: This study aims to identify the effect of the presence and absence of a ureteric stent on the outcome of ESWL in patients with proximal ureteric stones. Materials and Methods: This was a prospective cohort study done on 300 patients and wasdivided into two equal groups of 150 each. Group A patients were selected for ESWL with DJ stent, and Group B patients werefor ESWL without DJ stent. Noncontrast CT KUB was done as a functional study along with plainX-ray KUB and ultrasound before the intervention. All treatments were done with Direx Compact XL (electromagnetic generator) device as an outpatient procedure. Patients were followed up radiographically at 15 days, 1 month, 2 months, and in 3 months. Hematuria, steinstrasse, fever, ureteric colic, lower urinary tract symptoms (LUTS), stone clearance, number of ESWL sessions, and secondary procedures were recorded for each group. DJ stent was taken out when the stone disappeared or at the end of 3 months. Variables were compared with Chi-square test.Results: The overall stone-free rate at 3 months was $90.6 \%(272 / 300)$. Clearance after the first sitting, second sitting, and the third sitting wasnot significant between the two groups $(P>0.05)$. There was no significant difference between the two groups in regard to clearance according to the size of the stones. A total of $9.3 \%(28 / 300)$ of patients did not have a successful outcome and were termed as ESWL failures. Post- ESWL complications were significant between the both groups in terms of hematuria and ureteric colic. All the ureteric colic patients had a stone size larger than $13 \mathrm{~mm}, \mathrm{P}=0.0005$, which was more in the non-stented (Group B) patients. Most of the lower urinary tract symptoms were seen in stented (Group A) patients, $\mathrm{P}<0.0001$ significant between the two groups, and dysuria was also significant between the two groups with $P=0.0033$. Conclusion: Pre-ESWL ureteral stenting provides no additional benefit over no stent in situ, although ureteral stents are associated with more irritative symptoms.

Key words: DJ stent; Extracorporeal shock wave lithotripsy; Non-contrast computed tomography scan; Proximal ureteric stones

\section{INTRODUCTION}

The goal of treating proximal ureteric calculi is to achieve complete stone clearance with minimal morbidity for the patient. Since its introduction in the year 1980,
http://nepjol.info/index.php/AJMS DOI: 10.3126/ajms.v13i3.38918

E-ISSN: 2091-0576

P-ISSN: 2467-9100

Copyright (c) 2022 Asian Journal of Medical Sciences

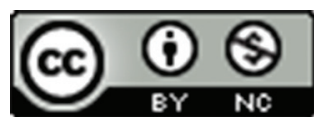

This work is licensed under a Creative Commons Attribution-NonCommercial 4.0 International License. 
stone fragmentation, laparoscopic method, and further improvements in ESWL have resulted in changes concerning the use of treatment modalities for ureteric stones. ${ }^{2}$ The success of ESWL has been correlated with the radiodensity of the stone on the plain X-ray KUB. ${ }^{2}$ ESWL is the minor invasive treatment for calculi of the upper urinary tract, and it is recommended as the first-line therapy. ${ }^{3}$ The density of stone measured by non-contrast computed tomography scan (NCCT), stone Hounsfield unit (HU) varies with the composition of the stone and determines the fragility of a calculus that ultimately governs the clinical outcome in ESWL.NCCT, because of its easy availability, has high sensitivity and very high-resolution capability is a promising modality for measuring stone density. ${ }^{3} \mathrm{ESWL}$ has a variable success rate for proximal ureteric calculi. ${ }^{4,5}$ Development of the small flexible ureterorenoscopy combined with advancements in intracorporeal lithotripters and lasers hasincreased the success rate for managing proximal ureteric calculi. However, a flexible ureteroscope is expensive and technique dependent.

Moreover, the current trend in surgery is toward minimally invasive procedures. The role of stents for ESWL in specific renal and ureteral stones remains controversial. The primary benefit of stents is to prevent complications associated with ureteric obstruction as stone fragments pass down the ureter. The drawbacks associated with stents are irritative symptoms and bladder discomfort in addition to risks of stent migration, vesicoureteral reflux, and stent encrustation. ${ }^{6}$ The effect of the ureteric stent on the outcome of ESWL continues to pose a dilemma, with no clear published recommendations. However, the use of stents is unavoidable in some cases (e.g., obstruction and sepsis).The overall accuracy of predicting calculi composition from plain radiographs was reported to be only $39 \%$, which is insufficient for clinical use. The emergence of a NCCT in assessing flank pain and the availability of the attenuation coefficient measurement has made many authors comparing attenuation and stone composition in vitro. ${ }^{7}$ Hence, with this background, the present study was conducted with an aim to identify the effect of the presence and absence of a ureteric stent on the outcome of ESWL in patients with proximal ureteric stones.

\section{Aims and objectives}

The aim of this study is to identify the effect of the presence of a ureteric stent on the outcome of extracorporeal shockwave lithotripsy, by comparing patients with proximal ureteric stones and to find out complications of extracorporeal shock wave lithotripsy during the management of proximal ureteric calculus.

\section{MATERIALS AND METHODS}

This was a prospective interventional study conducted in the department of urology at our instituteover a period of 3 years on patients who reported for the management of proximal ureteric calculus. All the patients were explained about the study, and consent was taken. The institutional ethical clearance was obtained for the study.

All the patients were briefed about the available methods of treatments and their complications in the management of upper ureteric calculus. These were medical expulsion therapy, ureteroscopy with intracorporeal lithotripsy, extracorporeal lithotripsy, percutaneous nephrolithotomy, and open surgery.

A detailed history and a thorough clinical examination were performed, followed by investigations including complete blood count, blood sugar, urea, serum creatinine, complete urine routine including urine culture, and sensitivity were done in all patients. A plain X-ray KUB and ultrasound were done in all patients. Either intravenous urography or non-contrast CT KUB was done as a functional study.

Inclusion criteria werepatients with unilateral upper ureteric calculus willing for extracorporeal shockwave lithotripsy, patients with normal renal parameters, no previous treatments for the same ureteric calculus, and no anatomical anomalies in the urinary tract. Exclusion criteria werepatients not willing for ESWL, bilateral ureteric calculi, coagulation disorder/patients on anticoagulation drugs, pregnancy, sepsis, and end-stage renal disease.

Three hundred patients were selected and randomly divided into two groups of 150 patients in each group. Group A patients were selected for ESWL with DJ stent and Group B for ESWL without DJ stent. In Group A patients, a prophylactic injection of gentamycin $80 \mathrm{mg}$ IM was given $1 \mathrm{~h}$ before the insertion of DJ stent, and then, a $5 \mathrm{Fr}$ $26 \mathrm{~cm} \mathrm{DJ} \mathrm{stent} \mathrm{was} \mathrm{placed} \mathrm{under} \mathrm{regional} \mathrm{anesthesia} \mathrm{before}$ ESWL. Bowel preparation with laxatives and anti-flatulence was given a day before the procedure. All treatments were done with Direx Compact XL (electromagnetic generator) device as an outpatient procedure(Figures 1 and 2). Stone was localized with fluoroscopy, and about 2500 shockwaves were given. The energy intensity was adjusted between 4 and 5 , and the shockwave rate was $60 / \mathrm{min}$. After each treatment session, patients were observed for $2-3 \mathrm{~h}$ and allowed to go home. Patients were explained about the post-treatmenthematuria, dysuria, and passage of stone fragments in the urine. Patients were advised to maintain adequate oral fluids. Patients were followed up at 15 days, 1 month, 2 months, and in 3 months or whenever patients had urinary complaints after the procedure. Failure was 
defined by the presence of fragments of any size in the follow-up film 3 months after the final ESWL session. ${ }^{8}$ Although history, physical examination, ultrasound KUB, and X-ray KUB weremade during the visits, a plain X-ray film was used as the standard method to identify any residual fragments. The treatment protocol included the second session of ESWL and, if necessary, the third session of ESWL. The patients follow-up was stopped if the patient cleared the stone with ESWL, or an auxiliary treatment was selected for the failure of ESWL.

\section{Outcome measurement}

\section{Primary outcome}

If a stone was not fragmented or if there was residual fragments $4 \mathrm{~mm}$ or larger after ESWL, this was considered as failure and auxiliary treatment option was sought. Secondary outcome-complications related to ESWL and DJ stenting such ashematuria, renal colic, fever, steinstrasse (stone street on follow-up radiography), stone clearance, number of ESWL sessions, LUTS, and secondary procedures were recorded for each group.

Lower urinary tract symptoms include increased frequency, urgency,nocturia, and burning sensation (dysuria) during micturition. It was evaluated in both groups by history during the follow-up period. Patients with complications

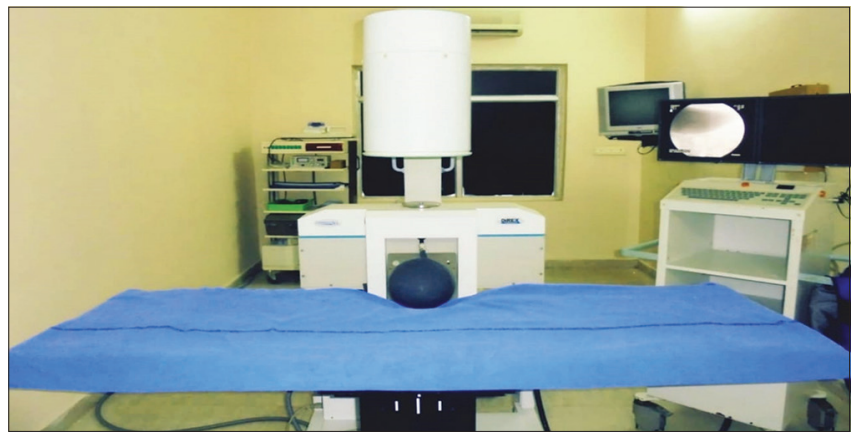

Figure 1: Direx Compact XL (electromagnetic generator) ESWL machineESWL: Extracorporeal shock wave lithotripsy

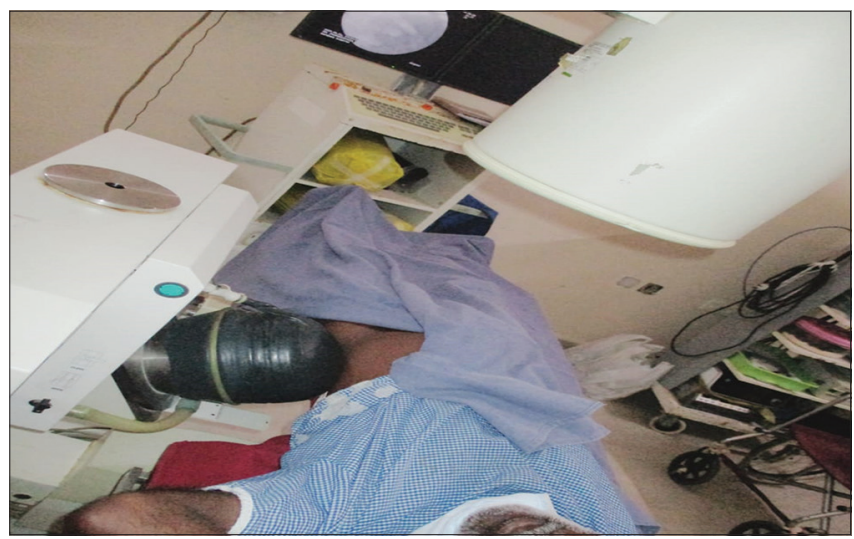

Figure 2: Stone localization with fluoroscopy and shock waves generation with electromagnetic generator like fever were treated with culture-specific antibiotics and antipyretics. The DJ stent was taken out when the stone disappeared, or at the end of 3 months, whichever is earlier.

\section{Statistical analysis}

The SPSS Version 17 package was employed to find Pearson's Chi-square test and Fisher's exact Chi-square test for the statistical analysis. $\mathrm{P}<0.05$ was considered as statistically significant.

\section{RESULTS}

The mean age of the patients in the study was 45.4 years. Mean age in Group A was 43.8 years and in Group B was 46.4 years ( $\mathrm{P}=0.48$ not significant). There were 114 male and 36 female patients in Group A and 118 males and 32 females in Group B ( $\mathrm{P}=0.581$ not significant) (Table 1).

The left side stones predominated over right-sided stones in both GroupsA and B. Patients with a stone size of $8-13 \mathrm{~mm}$ were 100 patients in each group, and patients of stone size between 14 and $19 \mathrm{~mm}$ were 50 patients in each group (Table 2).

In our study, overall stone-free rate at 3 months was $90.6 \%$ $(272 / 300)$. Clearance after the first sitting was $56.3 \%$ $(169 / 300)$, after the second sitting was $29.0 \%(87 / 300)$,

\begin{tabular}{|c|c|c|c|}
\hline Gender & $\begin{array}{l}\text { Stented } \\
\text { (Group A) }\end{array}$ & $\begin{array}{c}\text { Non-stented } \\
\text { (Group B) }\end{array}$ & $P$ value \\
\hline Males (n) & 114 & 118 & $0.581^{*}$ \\
\hline Females (n) & 36 & 32 & \\
\hline $\begin{array}{l}\text { Mean age } \\
\text { of patients }\end{array}$ & 43.8 years & 46.4 years & $0.48^{*}$ \\
\hline
\end{tabular}

*Based on Student's two-sample t-tests

\begin{tabular}{|c|c|c|c|}
\hline Variable & Stented & Non-stented & Total \\
\hline \multicolumn{4}{|l|}{ Side } \\
\hline Right & 71 & 73 & $144(48 \%)$ \\
\hline Left & 79 & 77 & $156(52 \%)$ \\
\hline \multicolumn{4}{|c|}{ Size of the stone } \\
\hline 8-10 mm & 45 & 45 & $90(30.0 \%)$ \\
\hline $11-13 \mathrm{~mm}$ & 55 & 55 & $110(36.6 \%)$ \\
\hline $14-16 \mathrm{~mm}$ & 35 & 35 & 70 ( $23.3 \%)$ \\
\hline $17-19 \mathrm{~mm}$ & 15 & 15 & $30(10.0 \%)$ \\
\hline \multicolumn{4}{|c|}{ Number of sittings } \\
\hline One & $79(52.6 \%)$ & $90(60.0 \%)$ & $169(56.3 \%)$ \\
\hline Two & $41(27.3 \%)$ & $46(30.6 \%)$ & $87(29.0 \%)$ \\
\hline Three & $11(7.3 \%)$ & $5(3.3 \%)$ & $16(5.3 \%)$ \\
\hline \multicolumn{4}{|c|}{ Complications } \\
\hline Yes & 31 & 27 & 58 \\
\hline No & 119 & 123 & 242 \\
\hline
\end{tabular}


and after the third sitting was 5.3\% (16/300) with $\mathrm{P}>0.05$ not significant between the two groups.

The patients were categorized according to stone size and the number of sittings they underwent in Table 3. There was no significance between the both groups.

A total of $9.3 \%(28 / 300)$ of patients did not have a successful outcome and were termed as ESWL failures. They required auxiliary the form of procedures in ureteroscopy with lithotripsy and stone extraction.

Post-ESWL complications consisted of 17 (5.6\%) patients with hematuria, fever in $9(3 \%)$ patients who were treated with urine culture-specific antibiotics, antipyretics and responded well,steinstrasse (the accumulation of ureteric stone fragments causing a ureteric blockage) in 17(5.6\%) patients, and ureteric colic requiring hospital admissions in $15(5 \%)$ patients. All the colic patients had a stone size larger than $13 \mathrm{~mm}$. $\mathrm{P}=0.0005$ is significant between the two groups (Table 4).

Among the lower urinary tract symptoms (LUTS), the frequency wasseen in $75(25 \%)$ cases, urgency in $84(28 \%)$ cases, dysuria in 109 (36\%) cases, and nocturia in 21 (7\%) cases. Most of the lower urinary tract symptoms were seen in stented (Group A) patients, $\mathrm{P} \leq 0.0001$ significant between the two groups and dysuria where $\mathrm{P}=0.0033$, which is significant (Table 5).

\section{DISCUSSION}

We had an overall stone-free rate of $90.6 \%$. This result compares favorably with previouslypublished series and is a timely reminder that a reasonable stone-free rate can be achieved without ureteroscopic use. The previous studies with different kind of lithotriptors reported success rates between $80 \%$ and $90 \% .^{7}$ In the study of Gnanapragasam et al., ${ }^{8}$ stone-free rates for upper ureteric stones were $90 \%$. Failure of ESWL was seen in patients with stone size $>1.3 \mathrm{~cm}$. Similarly, Mogensen and Anderson ${ }^{9}$ reviewed outcomes of 199 patients with ureteric stones treated with ESWL. Stone-free rates at the $3^{\text {rd }}$ and $6^{\text {th }}$ months after ESWL for upper ureteric stones were $86 \%$ and $91 \%$, respectively.

Hofbauer et al., ${ }^{10}$ evaluated the outcome of 1259 ureteric stones, with the success rate of upper ureteric stones being $98 \%$. We had a retreatment rate of $59 \%$, and additional procedures were required in $9.3 \%$ of cases. The American Ureteral Stones Clinical Guidelines Panel reported that, for proximal ureteric stones, the success rate of ESWL was $87 \%$ for $<1 \mathrm{~cm}$ stone and $76 \%$ for $>1 \mathrm{~cm}$ stone. In our study, almost the success rate was $95 \%$ in cases with

\begin{tabular}{|c|c|c|c|c|}
\hline Variable & Stented & Non-stented & Chi-square value & $P$ value \\
\hline \multicolumn{5}{|l|}{ Side } \\
\hline Right & 71 & 73 & 1.66 & 0.86 \\
\hline Left & 79 & 77 & & \\
\hline \multicolumn{5}{|c|}{ Number of sittings } \\
\hline One & 79 & 90 & 2.88 & 0.66 \\
\hline Two & 41 & 46 & & \\
\hline Three & 11 & 5 & & \\
\hline \multicolumn{5}{|c|}{ Stone size versus $1^{\text {st }}$ sitting } \\
\hline $8-10 \mathrm{~mm}$ & 34 & 39 & 2.39 & 0.72 \\
\hline $11-13 \mathrm{~mm}$ & 35 & 38 & & \\
\hline $14-16 \mathrm{~mm}$ & 09 & 11 & & \\
\hline $17-19 \mathrm{~mm}$ & 01 & 02 & & \\
\hline \multicolumn{5}{|c|}{ Stone size versus $2^{\text {nd }}$ sitting } \\
\hline $8-10 \mathrm{~mm}$ & 8 & 6 & 0.764 & 0.83 \\
\hline $11-13 \mathrm{~mm}$ & 11 & 14 & & \\
\hline $14-16 \mathrm{~mm}$ & 16 & 18 & & \\
\hline $17-19 \mathrm{~mm}$ & 06 & 08 & & \\
\hline \multicolumn{5}{|c|}{ Stone size versus $3^{\text {rd }}$ sitting } \\
\hline $8-10 \mathrm{~mm}$ & 02 & 0 & 1.531 & 0.88 \\
\hline $11-13 \mathrm{~mm}$ & 02 & 01 & & \\
\hline $14-16 \mathrm{~mm}$ & 03 & 02 & & \\
\hline $17-19 \mathrm{~mm}$ & 04 & 02 & & \\
\hline \multicolumn{5}{|c|}{$\begin{array}{l}\text { Stone-free rate according to } \\
\text { stone size }\end{array}$} \\
\hline $8-10 \mathrm{~mm}$ & 43 & 45 & 0.0355 & 0.91 \\
\hline $11-13 \mathrm{~mm}$ & 48 & 53 & & \\
\hline $14-16 \mathrm{~mm}$ & 29 & 31 & & \\
\hline $17-19 \mathrm{~mm}$ & 11 & 12 & & \\
\hline
\end{tabular}




\begin{tabular}{|c|c|c|c|c|}
\hline Variable & Stented & $\begin{array}{c}\text { Non- } \\
\text { stented }\end{array}$ & $\begin{array}{c}\text { Chi-square } \\
\text { value }\end{array}$ & $P$ value \\
\hline $\begin{array}{l}\text { Hematuria } \\
\text { (17) }\end{array}$ & 14 & 3 & 7.54 & 0.006 \\
\hline Fever (9) & 7 & 2 & 2.86 & 0.22 \\
\hline $\begin{array}{l}\text { Steinstrasse } \\
\text { (17) }\end{array}$ & 9 & 8 & 0.06 & 0.94 \\
\hline $\begin{array}{l}\text { Ureteric } \\
\text { colic (15) }\end{array}$ & 1 & 14 & 11.85 & 0.0005 \\
\hline
\end{tabular}

$\mathrm{P}<0.05$ was considered as statistically significant

\begin{tabular}{lcccc}
\multicolumn{5}{l}{ Table 5: The incidence of LUTS in both groups } \\
\hline Variable & Stented & $\begin{array}{c}\text { Non- } \\
\text { stented }\end{array}$ & $\begin{array}{c}\text { Chi-square } \\
\text { value }\end{array}$ & P value \\
\hline $\begin{array}{l}\text { Frequency } \\
(75)\end{array}$ & 64 & 11 & 49.93 & $<0.0001$ \\
$\begin{array}{l}\text { Urgency } \\
(84)\end{array}$ & 69 & 15 & 48.21 & $<0.0001$ \\
$\begin{array}{l}\text { Nocturia } \\
\begin{array}{l}(21) \\
\text { Dysuria } \\
(109)\end{array}\end{array}$ & 17 & 4 & 57.19 & $<0.0001$ \\
\hline
\end{tabular}

$\mathrm{P}<0.05$ was considered as statistically significant.LUTS: Lower urinary tract symptom

$<1 \mathrm{~cm}$ stone, while the success of $85 \%$ seen in $>1 \mathrm{~cm}$ stone. This rate of success may be due to better localization of the stone and the use of standard lithotripter (Direx Compact XL). The pushback technique of the stone was not used in our patients. All stones were treated without the manipulation of the stone. There is not much significant difference in success rates for in situ versus pushback ESWL. ${ }^{11}$ Macroscopic expansion space is not required for the successful fragmentation of ureteric calculi. ${ }^{12}$ Ureteral manipulations using the pushback technique are associated with a $5.1 \%$ perforation rate. We also observed that the presence of DJ stents significantly reduces the success rates.DJ stents were inserted in 150 cases preoperatively, of which $19(6 \%)$ patients required auxiliary procedure in the form of ureteroscopy. Ryan et al., ${ }^{13}$ showed that in situ ureteric stents impair ureteric peristalsis and trap large fragments, thus delaying stone clearance. The presence of a DJ stent next to the stone may prevent the full impact of the shock wave on the stone.

Several authors have attempted to identify the predictive factors associated with the failure of ESWL treatment for ureteric stones. Abdel-Khalek et al., ${ }^{14}$ in a study of 938 patients, defined a transverse stone diameter of $>10 \mathrm{~mm}$, site of stone, and presence of a stent as predictors of failure of ESWL. Kim et al., ${ }^{15}$ in a study of 369 patients, investigated factors that influence fragmentation of ureteric stones, reported as such the size of stone, radio-opacity, and degree of obstruction, but not the location. A study by Pareek et al., ${ }^{16}$ identified increased body mass index (BMI) and a high HUs value as independent predictors of the results of ESWL for upper urinary tract stones and devised an equation to compute the probability of treatment failure, which was $1 / 1+2.7(-z)$, where $Z=0.294$, BMI +0.13 , $\mathrm{HU}-18.98$.

In the context of stone disease, ureteric stents are usually inserted in cases of obstruction in a solitary kidney, patients with obstruction and fever at risk of sepsis, prolonged pain, and to prevent deterioration of renal function. Furthermore, their use is common in cases where large stones(usually $>20 \mathrm{~mm}$ ) are treated by ESWL and for managing steinstrasse and obstruction after ESWL. Bierkens et al., ${ }^{17}$ randomized 64 patients with significant renal stones(but no ureteric stones) and found a difference in the stone-free rate in 3 months of $9 \%$ in favor of the stented population( $44 \%$ vs. $35 \%$ ), while Pryor and Jenkins ${ }^{18}$ found a difference of $18 \%$ in the stone-free rate in favor of the unstented patients with ureteric stones. El-Assmy et al., ${ }^{19}$ randomized 186 patients with ureteric stones and moderate/severe hydronephrosis, and the results were better but not statistically significant for the unstented patients ( $91 \%$ vs. $85 \%$ stone-free rate, $\mathrm{P}=0.25)$. In comparison, Musa ${ }^{20}$ in 120 patients with renal stones found a stone-free rate of $91 \%$ versus $88 \%$ in favor of the unstented population and found a slightly higher incidence of fever in stented patients. This could explain the fact that patients with stent had two additional procedures performed, and a foreign body was placed in a sterile system.

Based on our findings and results of the previous studies, pre-SWL ureteral stenting does not appear to have definite advantages in terms of SFR or complications, compared to in situ SWL.

In addition, the decision to proceed with ureteral stenting requires much caution because it is a relatively invasive procedure. Furthermore, we should also consider the possibility of stent-related voiding symptoms, such as bladder irritation symptoms and flank pain or discomfort. ${ }^{21}$ In their randomized control study, Ghoneim et al., ${ }^{22}$ noted that microscopic hematuria, pyuria, dysuria, and suprapubic pain were significantly more common in patients with a ureteral stent than in those without. Another study, by ElAssmy et al., ${ }^{19}$ of patients with ureteral stones $2 \mathrm{~cm}$ or less causing moderate or severe obstruction also showed that the rates of post-SWL morbidities related to ureteral stents (such as suprapubic pain, gross or microscopic hematuria, pyuria, and positive urine cultures) were significantly higher in their ureteral stenting group. Thus, we recommend against routine pre-SWL ureteral stenting; instead, ureteral stents should be reserved for special indications, such as complicated urinary tract infection or severe pain. 
In a study by Madbouly et al., ${ }^{23}$ in the risk factors for forming a steinstrasse after ESWL, the overall incidence of steinstrasse was $3.97 \%$. Stone size and site, renal morphology, and shock wave energy are the significant predictive factors controlling steinstrasse formation. In our study, the incidence of steinstrasse was $5.67 \%$, and it was not significant between the two groups. If a patient has a high probability of steinstrasse formation, close follow-up with early intervention or prophylactic pre-ESWL ureteral stenting is indicated.

Most ESWL complications are minor and self-limiting, such as transient hematuria, pain, nausea, and vomiting; there are also life-threatening case reports described in the literature. In a study by Mohayuddin et al., ${ }^{24}$ the LUTSs, for example, urinary frequency, urgency, nocturia, dysuria, and hematuria were quite high in the stented group $(45 \%, 12.5 \%, 47.5 \%, 57.5 \%$, and $92.5 \%)$ as compared to the non-stented group $(7.5 \%, 2.5 \%, 10 \%, 15 \%$, and $67.5 \%$ ), respectively. These similar findings were noted in the other studies, for example, Preminger et al., ${ }^{25}$ found a higher incidence of LUTS in patients with stents than in the control group (43\% vs. $25 \%$ ). Similarly, in Chandoke et al., ${ }^{26}$ study, frequency, urgency, and dysuria were higher in the stented group. The same finding was reported by Musa who found a much higher frequency of LUTS (85\%) in the stented group as compared to the non-stentedgroup. ${ }^{20}$ It was suggested that the stent-related LUTSs were due to the presence of a foreign body in the urinary bladder irritating the trigone and the bladder neck. Ghoneim et al., ${ }^{22}$ in a study with 60 patients, concluded that no significant difference statistical difference was observed in stone-free rate between the stented and non-stented groups. Still, patients in the stented group had significant side effects, predominantly dysuria, urgency, frequency, and suprapubic pain attributed to the stent.

In our study, the stented group of patients had lower urinary tract symptoms than the non-stented group of patients. The negative effects of stenting may be explained by twotheories, which are not mutually exclusive. First, to have a maximal effect, shock waves must impinge on a stone surrounded by liquid.

The ureteral stent may absorb some of the energy created by the shock waves, thus reducing their effect on the stones. ${ }^{26}$ Second, the presence of a ureteral stent may cause ureteral edema, thus interrupting the passage of stone fragments. ${ }^{27}$ The impact would be greater with ureteral stones than renal stones because the area of contact between the stone and stent would be larger and ureteral edema would have a greater effect on ureteral stones.

\section{Limitations of the study}

The limitations of this study was patients with renal stones were excluded. We used a plain KUB film to determine whether patients were stone free, it is important to note that sometimes stones can be missed on plain KUB film. However, due to the study design, we considered all the cases with positive findings at X-ray KUB as residual stones.

\section{CONCLUSION}

Treatment with ESWL has low morbidity and high effectiveness. Pre-ESWL ureteral stenting provides no additional benefit over no stent in situ. Ureteral stents are associated with significant patient discomfort and morbidity. Although ureteral stents are associated with more irritative symptoms, their use resulted in less ureteric colic pain compared to no stent in situ when treated for proximal ureteric calculus.

\section{ACKNOWLEDGMENT}

We extend their sincere thanks to all the patients who participated in this study.

\section{REFERENCES}

1. Anagnostou T and Tolley D. Management of ureteric stones. Eur Urol. 2004;45(6):714-721. https://doi.org/10.1159/000475300

2. Segura JW, Preminger GM, Assimos DG, Dretler SP, Kahn RI, Lingeman JE, et al. Ureteral stones clinical guidelines panel summary report on the management of ureteral calculi. The American urological association. J Urol. 1997;158(5):19151921.

https://doi.org/10.1016/s0022-5347(01)64173-9

3. Blandy JP and Singh M. The case for a more aggressive approach to staghorn stones. J Urol. 1976;115(5):505-506. https://doi.org/10.1016/s0022-5347(17)59258-7

4. Liong ML, Clayman RV, Gittes RF, Lingeman JE, Huffman JL and Lyon ES. Treatment options for proximal ureteral urolithiasis: Review and recommendations. J Urol. 1989;141(3):504-509. https://doi.org/10.1016/s0022-5347(17)40874-3

5. Grasso M, Beaghler M and Loisides P. The case for primary endoscopic management of upper urinary tract calculi: II. Cost and outcome assessment of 112 primary ureteral calculi. Urology. 1995;45(3):372-376. https://doi.org/10.1016/S0090-4295(99)80003-X

6. Saltzman B. Ureteral stents: Indications, variation and complications. J Urol. 1989;141(5):1278-1278.

7. Mobley TB, Myers DA, Jenkins JM, Grine WB and Jordan WR. Effects of stents on lithotripsy of ureteral calculi: Treatment results with 18,825 calculi using the Lithostar lithotriptor. J Urol. 1994;152(1):53-56.

https://doi.org/10.1016/s0022-5347(17)32814-8

8. Gnanapragasam VJ, Ramsden PD, Murthy LS and Thomas DJ. Primary in situ extracorporeal shock wave lithotripsy in the 
management of ureteric calculi: results with a third-generation lithotripter: Extracorporeal shock wave lithotripsy. BJU Int. 1999;84(7):770-774.

https://doi.org/10.1046/j.1464-410x.1999.00284.x

9. Mogensen $\mathrm{P}$ and Anderson JT. Primary in situ extracorporeal shock wave lithotripsy for Ureteral calculi. Scand J Urol Nephrol Suppl. 1994;157:159-163.

10. Hofbauer J, Tuerk C, Höbarth K, Hasun R and Marberger M. ESWL in situ or ureteroscopy for ureteric stones? World J Urol. 1993;11(1):54-58.

https://doi.org/10.1007/BF00182172

11. Danuser H, Ackermann DK, Marth DC, Studer UE and Zingg EJ. Extracorporeal shock wave lithotripsy in situ or after push-up for upper ureteral calculi: A prospective randomized trial. J Urol. 1993;150(3):824-826.

https://doi.org/10.1016/s0022-5347(17)35623-9

12. Netto NR, Lemos GC and Claro JF. In situ extracorporeal shock wave lithotripsy for ureteral calculi. J Urol. 1990;144(2 Part 1):253-254.

https://doi.org/10.1016/s0022-5347(17)39425-9

13. Ryan PC, Lennon GM, McLean PA and Fitzpatrick JM. The effects of acute and chronic $\mathrm{JJ}$ stent placement on upper urinary tract motility and calculus transit. Br J Urol. 1994;74(4):434-439. https://doi.org/10.1111/j.1464-410x.1994.tb00418.x

14. Abdel-Khalek M, Sheir K, Elsobky E, Showkey S and Kenawy M. Prognostic factors for extracorporeal shock-wave lithotripsy of ureteric stones--a multivariate analysis study. Scand J Urol Nephrol. 2003;37(5):413-418.

https://doi.org/10.1080/00365590310006255

15. Kim HH, Lee JH, Park MS, Lee SE and Kim SW. In situ extracorporeal shockwave lithotripsy for ureteral calculi: Investigation of factors influencing stone fragmentation and appropriate number of sessions for changing treatment modality. J Endourol. 1996;10(6):501-505.

https://doi.org/10.1089/end.1996.10.501

16. Pareek G, Armenakas NA, Panagopoulos G, Bruno JJ and Fracchia JA. Extracorporeal shock wave lithotripsy success based on body mass index and Hounsfield units. Urology. 2005;65(1):33-36.

https://doi.org/10.1016/j.urology.2004.08.004

17. Bierkens AF, Hendrikx AJ, Lemmens WA and Debruyne FM. Extracorporeal shock wave lithotripsy for large renal calculi: The role of ureteral stents. A randomized trial. J Urol. 1991;145(4):699-702. https://doi.org/10.1016/s0022-5347(17)38428-8

18. Pryor $\mathrm{JL}$ and Jenkins $A D$. Use of double-pigtail stents in extracorporeal shock wave lithotripsy. J Urol. 1990;143(3):475-478. https://doi.org/10.1016/s0022-5347(17)39993-7

19. El-Assmy A, El-Nahas AR and Sheir KZ. Is pre-shock wave lithotripsy stenting necessary for ureteral stones with moderate or severe hydronephrosis? J Urol. 2006;176(5):2059-2062; discussion 2062.

https://doi.org/10.1016/j.juro.2006.07.022

20. Musa AA. Use of double-J stents prior to extracorporeal shock wave lithotripsy is not beneficial: Results of a prospective randomized study. Int Urol Nephrol. 2008;40(1):19-22. https://doi.org/10.1007/s11255-006-9030-8

21. Abt D, Mordasini L, Warzinek E, Schmid HP, Haile SR, Engeler DS, et al. Is intravesical stent position a predictor of associated morbidity? Korean J Urol. 2015;56(5):370-378. https://doi.org/10.4111/kju.2015.56.5.370

22. Ghoneim IA, El-Ghoneimy MN, El-Naggar AE, Hammoud KM, El-Gammal MY and Morsi AA. Extracorporeal shock wave lithotripsy in impacted upper ureteral stones: A prospective randomized comparison between stented and non-stented techniques. Urology. 2010;75(1):45-50. https://doi.org/10.1016/j.urology.2009.06.071

23. Madbouly K, Sheir KZ, Elsobky E and Eraky I. Risk factors for the formation of a steinstrasse after extracorporeal shock wave lithotripsy: A statistical model. J Urol. 2002;167(3):1239-1242.

24. Mohayuddin N, Malik HA, Hussain M, Tipu SA, Shehzad A, Hashmi A, et al. The outcome of extracorporeal shockwave lithotripsy for renal pelvic stone with and without $\mathrm{JJ}$ stent--a comparative study. J Pak Med Assoc. 2009;59(3):143-146.

25. Preminger GM, Kettelhut MC, Elkins SL, Seger J and Fetner CD. Ureteral stenting during extracorporeal shock wave lithotripsy: help or hindrance? J Urol. 1989;142(1):32-36. https://doi.org/10.1016/s0022-5347(17)38654-8

26. Chandhoke PS, Barqawi AZ, Wernecke $C$ and Chee-Awai RA. A randomized outcomes trial of ureteral stents for extracorporeal shock wave lithotripsy of solitary kidney or proximal ureteral stones. J Urol. 2002;167(5):1981-1983.

27. Joshi HB, Obadeyi $\mathrm{OO}$ and Rao PN. A comparative analysis of nephrostomy, JJ stent and urgent in situ extracorporeal shock wave lithotripsy for obstructing ureteric stones. BJU Int. 1999;84(3):264-269. https://doi.org/10.1046/j.1464-410x.1999.00174.x

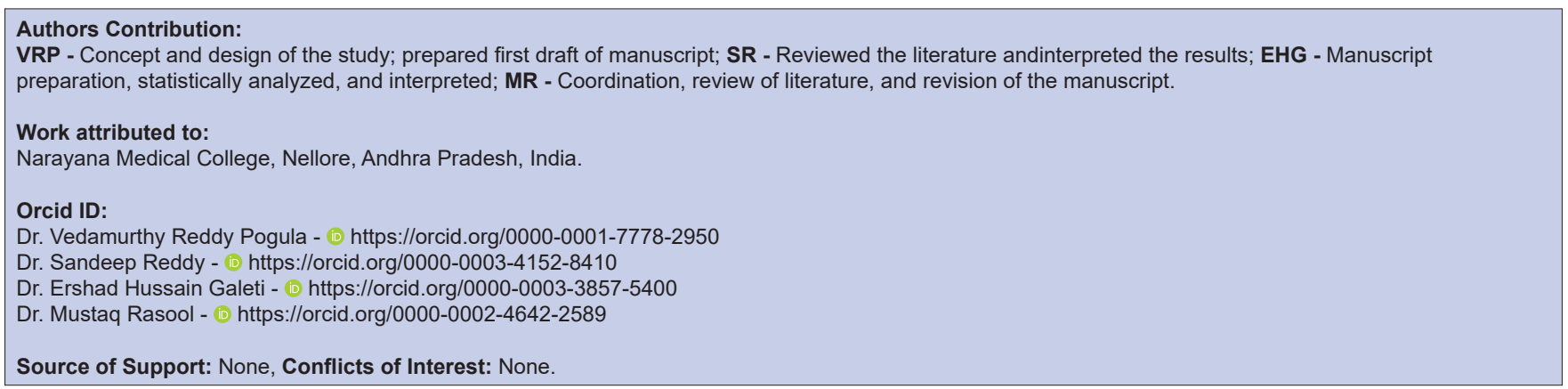

